\title{
Percepción de la población respecto a los riesgos asociados a los estudios de imágenes
}

Drs. M. Carolina Carvajal B(1) Roberto Vallejo $\mathrm{P}^{(1)}$, Rodrigo Bazaes $C^{(2)}$, Cristián Varela $\mathrm{U}^{(3)}$, TM. Aquiles Zavala(4), Int. Daniel Alvarez $G^{(5)}$.

1. Residente de Radiología, Facultad de Medicina, Universidad de los Andes. Santiago - Chile.

2. Radiólogo, Profesor Asistente, Facultad de Medicina, Universidad de los Andes, Departamento de Radiología, Clínica Santa María. Santiago - Chile.

3. Radiólogo, Profesor Asistente, Facultad de Medicina, Universidad de los Andes- Universidad Mayor, Departamento de Radiología, Clínica Dávila. Santiago - Chile.

4. Tecnólogo Médico, Coordinador jefe, Departamento de Radiología, Clínica Dávila. Santiago - Chile.

5. Interno de Medicina, Séptimo año, Universidad de los Andes. Santiago - Chile.

Public perception of radiation-related risks of imaging studies

Abstract. Introduction: In recent years, diagnostic procedures using ionizing radiation have achieved a great boom, although patients are often unaware of the risks they involve. Objectives: To know how is ionizing radiation perceived by the population attending Clínica Dávila, Department of Radiology (DRCD). Methods: A voluntary and anonymous survey was conducted on patients and / or companions. Results: A total of 330 adults were surveyed. An $57 \%$ had completed higher education, whereas $7.9 \%$ had only finished elementary schooling; $78.8 \%$ had undergone three or more imaging tests. CT examination was considered to pose the highest levels of risk (42.4\%), followed by Ultrasonography (30.6\%). Discussion: The survey group was made up of young adults, mostly college graduates, and predominantly female. Surprinsingly enough, considering that obstetric sonography is commonly used during pregnancy, US was ranked second in terms of associated health risks. There is an evident interest in the subject, since $90 \%$ of responders would like to receive further information.

Key words: Adverse effects, Imaging, Patient medical education, Patient radiation dose, Public perception, Radiation risk.

Resumen. Introducción: Los procedimientos diagnósticos que usan radiación ionizante están en auge, aunque conllevan riesgos muchas veces desconocidos por los pacientes. Objetivos: Conocer la percepción de la población que acude al Departamento de Radiología de Clínica Dávila (DRCD) sobre la radiación. Material y métodos: Se aplicó una encuesta voluntaria y anónima a pacientes y/o acompañantes. Resultados: Se encuestó a 330 adultos. El 57\% poseía estudios superiores y 7,9\% sólo estudios básicos. El 78,8\% se había efectuado 3 o más estudios radiológicos. La TC fue el examen considerado con mayor riesgo (42,4\%), luego la US (30,6\%). Discusión: El grupo encuestado fueron adultos jóvenes, con estudios superiores en su mayoría y con predominio femenino. Sorprendió que la US se considerara como el segundo examen de mayor riesgo, ya que las mujeres poseen mayor contacto con este estudio durante el embarazo. Hay interés en el tema, porque el $90 \%$ desea recibir información.

Palabras clave: Dosis de radiación, Educación médica del paciente, Imagenología efectos adversos, Percepción social, Riesgo radiológico.

Carvajal MC, et al. Percepción de la población respecto a los riesgos asociados a los estudios de imágenes. Rev Chil Radiol 2012; 18(2): 80-84.

Correspondencia: Dra. M. Carolina Carvajal Benavides / mcarolinacarvajal@gmail.com

Trabajo recibido el 12 de julio de 2011, aceptado para publicación el 22 de mayo de 2012.

\section{Introducción}

En los últimos años, los procedimientos diagnósticos y terapéuticos que utilizan radiaciones ionizantes han alcanzado gran auge en la medicina moderna. Se ha estimado que en el mundo, durante el período 20002007, se efectuó anualmente un promedio de 3.600 millones de procedimientos médicos que utilizaron radiaciones ionizantes ${ }^{(1)}$. 
En radiología los estudios que requieren radiación ionizante son la radiografía $(\mathrm{Rx})$ y la tomografía computada (TC) (1).

Superado cierto nivel umbral, los riesgos son directamente proporcionales a la dosis recibida; mientras mayor cantidad de radiación los riesgos son más altos ${ }^{(2)}$.

Los efectos biológicos observados en personas irradiadas se clasifican en: a) Determinísticos, que son los efectos que aparecen a un umbral conocido de radiación, por ejemplo, las cataratas; y b) Estocásticos, corresponden a los efectos que en virtud del aumento de la dosis de exposición se incrementa la probabilidad de ocurrencia, sin embargo, dependen de otros factores de suceptibilidad biológica individual, por ejemplo, el potencial desarrollo de una neoplasia secundaria a partir de una mutación inducida por radiación ${ }^{(2)}$.

Precisamente esta última patología es una de las consecuencias más temidas por la población que se realiza un estudio radiológico. El riesgo potencial depende tanto del tipo como de la cantidad de estudios que se realicen, así como también de la suceptibilidad personal. Sólo algunos de estos factores son cuantificables en la actualidad ${ }^{(1,3)}$.

Con el aumento de la disponibilidad de los equipos radiológicos, el avance de las tecnologías, así como también el aumento de las indicaciones clínicas, los pacientes son sometidos a una mayor cantidad de radiación, que se ha ido acentuando especialmente en la última década ${ }^{(1,3)}$. Es por esto también que se ha generado la necesidad de entregar un consentimiento informado, con los potenciales riesgos y complicaciones, además de los detalles del procedimiento a efectuar ${ }^{(4)}$. Incluso algunas en la literatura recomiendan registrar la dosis histórica acumulada(5,6).

En vista de la trascendencia, tanto biológica como médico-legal, que posee la utilización de energía ionizante en radiodiagnóstico, pretendimos investigar mediante una sencilla encuesta la percepción del paciente acerca de los riesgos a los que está expuesto y determinar cuán cerca o lejos se encuentra la percepción de la realidad. Además, evaluamos el interés de los usuarios en adquirir mayor información respecto al tema.
Material y métodos

Durante los meses de mayo a julio de 2010 se aplicó una encuesta voluntaria y anónima a pacientes y acompañantes que acudieron al Departamento de Radiología de Clínica Dávila, acerca de los riesgos asociados a la radiación en los distintos estudios de imágenes que normalmente se realizan (ultrasonografía, radiografía, tomografía computada y resonancia magnética).

La encuesta se tituló "¿Corremos algún riesgo al hacernos exámenes radiológicos?" (Anexo 1), y constó de siete preguntas de selección múltiple más la solicitud de algunos datos demográficos (Tabla I). Esta fue realizada por un interno de medicina (DAG), quien entrevistó a todos los pacientes y/o acompañantes que se encontraban en la sala de espera del Departamento de Radiología de Clínica Dávila que quisieran participar del estudio, no importando el tipo de examen que se iban a realizar (muestreo por conveniencia).

Los datos recolectados se insertaron en una base de datos y se realizaron análisis porcentuales.

\section{Resultados}

Se encuestó a un total de 330 personas adultas con un promedio de edad de 42 años (rango: $18-81$ años), predominaron las personas de sexo femenino $(63,6 \%)$.

Un 39,4\% de los entrevistados correspondió a quien se le efectuaría el estudio, el resto fueron los acompañantes. El 57\% del total poseía algún estudio superior y el 7,9\% sólo tenía estudios básicos. Un $8,5 \%$ de los individuos encuestados trabajaba en el ámbito de la salud.

El 78,8\% se había efectuado tres o más estudios en un servicio de radiología.

El $42,4 \%$ consideró que la TC era el examen con mayor riesgo, en segundo lugar fue mencionada la ecografía (30,6\%). En relación al riesgo específico de una TC, el $43,9 \%$ la consideró de bajo riesgo y un $24 \%$ la consignó de alto riesgo, en relación a la radiografía que sólo el $13 \%$ la considera de alto riesgo (Tabla II).

Tabla I. Datos Demográficos.

\begin{tabular}{|lrlllr|} 
Edad & $\mathrm{n}^{\circ}$ & Sexo & $\%$ & Educación & $\mathrm{n}^{\circ}$ \\
\hline$<21$ & 13 & Femenino & $64,8 \%$ & Superior & 188 \\
$21-39$ & 125 & Masculino & $35,2 \%$ & Media & 107 \\
$40-65$ & 164 & & & Básica & 26 \\
$>65$ & 17 & & & No contesta & 9 \\
No contesta & 11 & & & & \\
\hline
\end{tabular}


Tabla II. Preguntas y respuestas.

\begin{tabular}{|c|c|c|c|c|}
\hline Preguntas & as $\left(n^{0}\right) \%$ & Preguntas & Respuestas $\left(\mathrm{n}^{\circ}\right) \%$ & \\
\hline \multicolumn{5}{|c|}{$\begin{array}{l}\text { De los siguiente exámenes, ¿cuál considera ud. que ¿Qué daño eventual a la salud le podría } \\
\text { posee mayor riesgo de provocar algún daño a la salud? } \quad \text { producir la toma de una radiografía? }\end{array}$} \\
\hline Ecografía & $30,6 \quad(101)$ & Alteración de la visión & 15,5 & $(51)$ \\
\hline Radiografía & $2,1 \quad$ (7) & Infertilidad & 14,5 & (48) \\
\hline Tomografía Computada & $42,4(140)$ & Malformaciones & 44,5 & (127) \\
\hline Resonancia Magnética & $20,6 \quad(68)$ & Otros & 12,4 & $(41)$ \\
\hline \multirow[t]{3}{*}{ No contesta } & 4,2 & Ninguno & 25,0 & $(7,6)$ \\
\hline & & No sabe & 2,4 & $(8)$ \\
\hline & & No contesta & 9,1 & (30) \\
\hline \multicolumn{2}{|l|}{$\begin{array}{l}\text { Si ud. se realiza una radiografía, el riesgo } \\
\text { de recibir algún daño es: }\end{array}$} & \multicolumn{3}{|c|}{ ¿Se ha efectuado estudios radiológicos previos? } \\
\hline Alto & 13,0 & $\mathrm{Si}$ & 88,2 & $(291)$ \\
\hline Bajo & 52,7 & No & 8,5 & $(28)$ \\
\hline Muy bajo & $16,4 \quad(54)$ & No contesta & 3,3 & (11) \\
\hline No existe & 15,2 & & & \\
\hline No contesta & $2,7 \quad(9)$ & & & \\
\hline \multicolumn{5}{|c|}{$\begin{array}{l}\text { Si ud. se realiza un scanner (TC sin contraste), } \\
\text { riesgo de recibir algún daño a la salud es: }\end{array}$} \\
\hline Alto & $24,5 \quad(81)$ & Sólo uno & 3,9 & (13) \\
\hline Bajo & 43,9 (145) & No contesta & 11,8 & (39) \\
\hline Muy bajo & $14,8 \quad(49)$ & & & \\
\hline No exite & $13,6 \quad(45)$ & ¿Le gustaría tener ma) & n sobre el tema? & \\
\hline \multirow{3}{*}{$\begin{array}{l}\text { No contesta } \\
\text { Si }\end{array}$} & $3,0 \quad(10)$ & & & \\
\hline & $90,6(299)$ & No & 5,5 & (18) \\
\hline & & No contesta & 3,9 & (13) \\
\hline
\end{tabular}

En relación con los potenciales daños de una radiografía, el 38,5\% cree que causa malformaciones, un $15,5 \%$ alteración de la visión y $2,4 \%$ no sabe.

Al $90,6 \%$ de los encuestados le gustaría recibir mayor información sobre el tema.

\section{Discusión}

El grupo encuestado correspondió a una población de personas adultas jóvenes, donde predominó el sexo femenino y la mayoría poseía algún estudio superior.

En relación a la pregunta respecto a qué estudio provoca mayor daño a la salud, la respuesta más elegida fue la TC y concuerda con el hecho de que es el examen que emplea mayor dosis de radiación, y por ello teóricamente podría significar mayor riesgo. A pesar de esto, se han efectuado varios estudios en donde se concluye que el riesgo poblacional es bajo ${ }^{(1)}$, pero es difícil de estimar el riesgo individual y se recomienda siempre evaluar la necesidad del estudio (costo/beneficio), ajustar la dosis en forma individual e informar al paciente (consentimiento) ${ }^{(4,5)}$.

La ecografía se consideró en segundo lugar, situación llamativa ya que las mujeres son las que poseen mayor contacto con este estudio, especialmente durante los embarazos, donde deberían haberse informado acerca de la seguridad y más aún considerando que la población estudiada fue predominantemente femenina y con algún estudio superior.

Ante la pregunta del riesgo por estudio especí fico, tanto para la TC como para la Rx predominaron las respuesta de bajo riesgo o inferior, alcanzando el $72,3 \%$ para la TC y el $84,3 \%$ para la $\mathrm{Rx}$, hecho que es avalado por la literatura, ya que la dosis empleada por estos estudios es muy baja y por ello los riesgos potenciales también son bajos.

A pesar de que son considerados de bajo riesgo, ante la pregunta de los daños eventuales a la salud que podría producir una radiografía, el 75\% eligió alguna consecuencia grave (alteración de la visión, infertilidad, malformación y otros), lo que habla de una percepción desproporcionada de los riesgos reales. Para graficar esto en la Tabla III se hacen estimaciones de la pérdida de expectativa de vida en actividades cotidianas comparadas con 
Tabla III. Pérdida estimada de expectativa de vida en actividades cotidianas.

Eventos PEV**

Fumar un cigarillo

$10 \mathrm{~min}$

Volar de costa a costa en EE.UU.

$100 \mathrm{~min}$

Accidentes en el hogar

95 días

Accidente automovilístico

207 días

1 mrad* $^{*}$ de radiación

1,5 minutos

**PEV: Pérdida de expectativa de vida

*mrad: dosis equivalente a dos radiografías de tórax

Adaptada de Ludwing R, Turner L. Public Perceptions of

Radiation Exposure Risk. J Allied Health. 2002 Fall; 31(3):

$159-164^{(7)}$. una dosis estándar de radiación que equivale a dos radiografías de tórax $(1 \mathrm{mrad})^{(7)}$.

A su vez, más de un tercio de los encuestados poseen tres o más exámenes en radiología, hecho que evidencia la gran demanda por los estudios radiológicos y no hace más que confirmar la importancia de referirnos a los riesgos que conlleva, sobretodo porque este número debería ir ascendiendo en un futuro cercano, planteando la inquietud de considerar un registro histórico de la dosis de radiación, tal como se efectúa en otros países.

Un hecho importante fue que al $90 \%$ de los encuestados les gustaría recibir mayor información y nos plantea el desafío como radiólogos de participar en esta educación.

Anexo 1.

Encuesta anónima: “Corremos algún riesgo al hacernos exámenes radiológicos?"

Paciente Acompañante

Sexo

$F \square \quad M$

Estudios

Básica $\square$

¿Trabaja Ud. en algo relacionado con la salud?

¿Se ha informado acerca de este tema?

Sí

Edad:

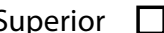

Sí $\square$ No

No

1.- De los siguientes exámenes, ¿cuál considera Ud. que posee mayor riesgo de provocar algún daño a la salud?
a. Ecografía
b. Radiografía
c. Scanner (sin contraste)
d. Resonancia magnética

2.- Si usted se realiza una radiografía, el riesgo de recibir algún daño a la salud es:
a. Alto
b. Bajo
c. Muy bajo
d. No existe

3.- Si usted se realiza un scanner (TC sin contraste), el riesgo de recibir algún daño a la salud es:
a. Alto
b. Bajo
c. Muy bajo
d. No existe

4.- ¿Qué daño eventual a la salud le podría producir la toma de una radiografía?
a. Alteraciones de la visión
b. Infertilidad
c. Malformaciones
d. Otros
Si eligió Otros, ¿cuál sería ese daño?

5.- ¿Se ha efectuado estudios radiológicos previos?

6.- En caso que su respuesta fue SÍ, ¿cuántos estudios se ha efectuado?
a. Sólo uno
b. Dos
c. Tres o más

7.- ¿Le gustaría tener mayor información sobre el tema?

Sí

No

Muchas gracias por su colaboración. 
Se debe reconocer que ésta fue una encuesta sencilla, en un grupo de población que no representa la demografía chilena y que deja la inquietud manifiesta de seguir investigando.

\section{Bibliografía}

1. Hall E, Brenner D. Cancer risks from diagnostic radiology. The British Journal of Radiology. 2008; 81: 362-378.

2. Bushong SC. Radiologic Science for Technologists. 8th ed. 2005: 480-581.

3. Brenner DJ, Hall EJ. Computed tomography-an increasing source of radiation exposure. $\mathrm{N}$ Engl J Med. 2007; 357(22): 2277-2284.

4. Lee $\mathrm{Cl}$, Flaster $\mathrm{HV}$, Haims $\mathrm{AH}$, Monico EP, Forman
HP. Diagnostic CT scans: institutional informed consent guidelines and practices at academic medical centers. AJR Am J Roentgenol. 2006; 187(2): 282-287.

5. Miller D, Kwon D, Bonavia G. Reference Levels for Patient Radiation Doses in Interventional Radiology: Proposed Initial Values for U.S. Practice. Radiology 2009; 253: 753-764.

6. Amis E, Butler P, Applegate K, Birnbaum K, Brateman L. Hevezi J, et al. American College of Radiology White Paper on Radiation Dose in Medicine. J Am Coll Radiol 2007; 4: 272-284.

7. Ludwig R, Turner L. Public Perceptions of Radiation Exposure Risk. J Allied Health. 2002 Fall; 31(3): 159164.

\section{Sப canocimienta está en nuestras manas}

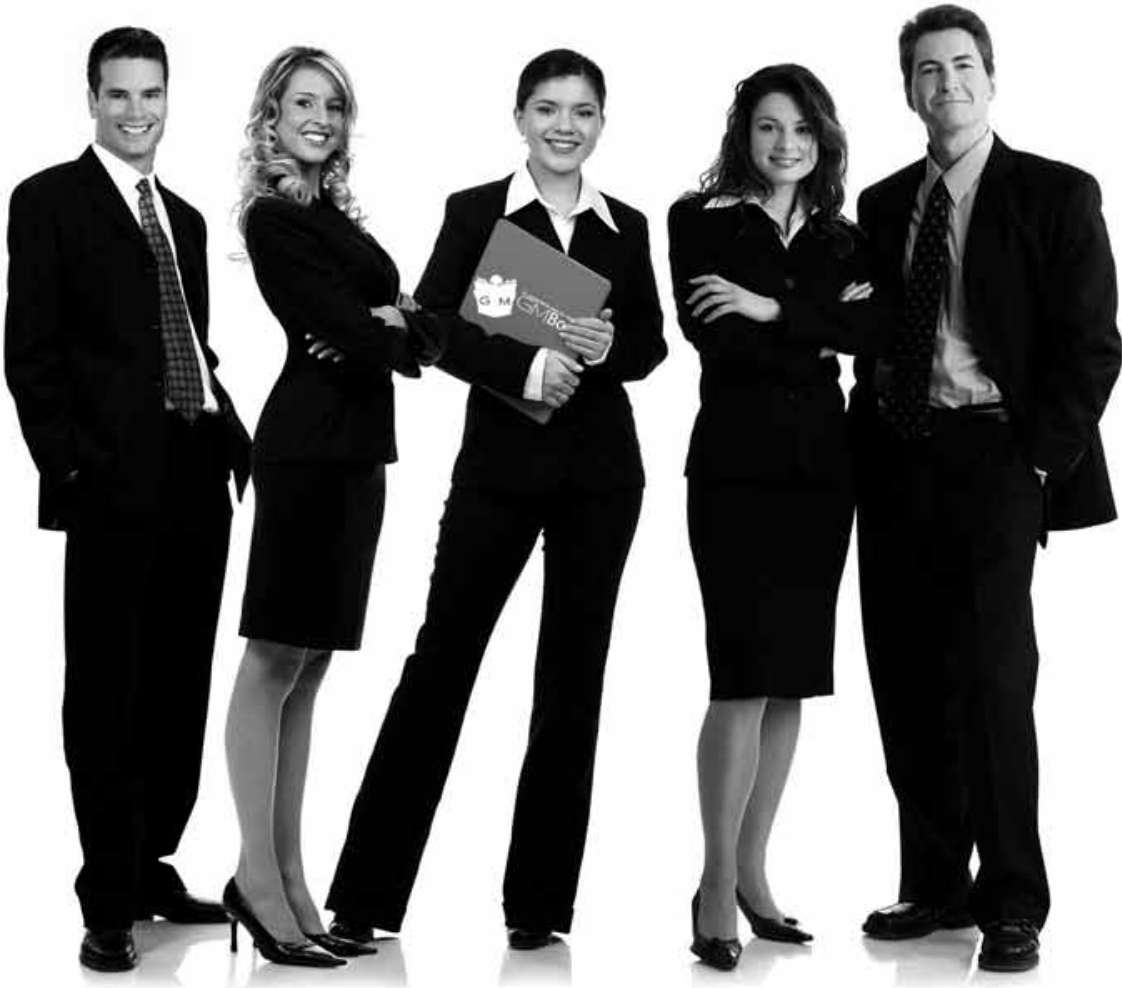

- Arquitectura

- Educación

- Derecho

- Medicina

- Psicología

Áreas

- Economía

- Contabilidad

- Informática

- Comunicación

- Turismo

- Ingeniería

- Arte

- Ciencias Sociales

Distribuidora de libros nacionales e internacionales 